\title{
Comparison of Precision between Optical and Electromagnetic Navigation Systems in Total Knee Arthroplasty
}

\author{
Seung Joon Rhee, MD, Shi Hwan Park, MD, He Myung Cho, MD, and Jeung Tak Suh, MD \\ Department of Orthopaedic Surgery, Pusan National University Hospital, Busan, Korea
}

\begin{abstract}
Purpose: The purpose of this study is to compare and analyze the precision of optical and electromagnetic navigation systems in total knee arthroplasty (TKA).

Materials and Methods: We retrospectively reviewed 60 patients who underwent TKA using an optical navigation system and 60 patients who underwent TKA using an electromagnetic navigation system from June 2010 to March 2012. The mechanical axis that was measured on preoperative radiographs and by the intraoperative navigation systems were compared between the groups. The postoperative positions of the femoral and tibial components in the sagittal and coronal plane were assessed.

Results: The difference of the mechanical axis measured on the preoperative radiograph and by the intraoperative navigation systems was 0.6 degrees more varus in the electromagnetic navigation system group than in the optical navigation system group, but showed no statistically significant difference between the two groups ( $p>0.05$ ). The positions of the femoral and tibial components in the sagittal and coronal planes on the postoperative radiographs also showed no statistically significant difference between the two groups ( $\mathrm{p}>0.05$ ).

Conclusions: In TKA, both optical and electromagnetic navigation systems showed high accuracy and reproducibility, and the measurements from the postoperative radiographs showed no significant difference between the two groups.
\end{abstract}

Keywords: Knee, Arthroplasty, Navigation, Optical and electromagnetic, Precision

\section{Introduction}

The goal of total knee arthroplasty (TKA) is to relieve pain and to improve quality of life by replacing the deformed knee joint. The success of TKA depends on various factors such as the patient selection, implant selection, soft-tissue balancing, restoration of the normal alignment of the lower limb and joint line, proper surgical technique, and preoperative and postoperative

Received August 16, 2013; Revised (1st) January 3, 2014;

(2nd) June 16, 2014; (3rd) August 4, 2014; Accepted August 7, 2014

Correspondence to: Jeung Tak Suh, MD

Department of Orthopaedic Surgery, Pusan National University

Hospital, 179 Gudeok-ro, Seo-gu, Busan 602-739, Korea

Tel: +82-51-240-7248, Fax: +82-51-247-8395

E-mail: jtsuh@pusan.ac.kr

This is an Open Access article distributed under the terms of the Creative Commons Attribution Non-Commercial License (http://creativecommons.org/licenses/by-nc/3.0/) which permits unrestricted non-commercial use, distribution, and reproduction in any medium, provided the original work is properly cited. management. In particular, accurate insertion of the implant and correction of lower limb alignment greatly affect the clinical outcome and life span of TKA. Inaccurate positioning of the implant causes early loosening of the implant or wear-off of polyethylene or loss of function. In particular, varus/valgus malalignment is known as a common cause of early loosening of the implant ${ }^{1-3)}$.

Accordingly, in order to insert implants in the accurate position and to have desirable mechanical alignment, diverse computerassisted navigation systems were recently developed. These systems include optical navigation system, electromagnetic navigation system, recently introduced ultrasonic wave-based navigation system, etc., which are reported to have a margin of error under $1 \mathrm{~mm}$ or $1^{04-6)}$.

However, comparative research on the precision of the optical navigation system and the electromagnetic navigation system is rare. Hence, in the present study, we aimed at exploring whether there is a difference in the precision and consistency between the optical and electromagnetic navigation systems. We hypothesized that the precision of the optical navigation system and electro- 
magnetic navigation system would be significantly different, and compared and analyzed measurements of both systems to confirm the hypothesis.

\section{Materials and Methods}

\section{Materials}

This was a prospective randomized study conducted from June 2010 and March 2012 at our institution. The criteria for inclusion included: 1) a diagnosis of primary degenerative osteoarthritis and 2) surgery on just one side; the criteria for exclusion included: 1) patients with mental disorder or mental illness, 2) severe contralateral knee osteoarthritis, 3) total hip arthroplasty (THA), contralateral TKA, or periprosthetic fractures in the period of study, 4) patients with symptomatic arthritis in the back, hip, contralateral knee, and feet, 5) a history of patella fracture, high tibial or distal femoral osteotomy, THA and TKA, and 6) a fixed varus, or valgus deformity of $>15^{\circ}$. A total of 134 patients were eligible for assessment, and 9 were excluded before randomization. There remained 125 (17 males and 108 females) patients. In the operating room, all of the patients were assigned to the OrthoSoft optical navigation system (Zimmer CAS, Montreal, QC, Canada) assisted group or the AxiEM electromagnetic navigation system (Medtronic Inc., Louisville, CO, USA) assisted group by a computer-generated randomization sequence. In order to protect the integrity of the randomization scheme, an independent biostatistician ensured that the block sizes were confidential (Fig. 1). The implant used in all cases of the two groups was LPS-Flex
(Nexgen system; Zimmer Inc., Warsaw, IN, USA) that is a highflexion TKA design. The mean age of the patients was 67.5 years (range, 62 to 78 years) in the optical navigation system group and 68.5 years (range, 60 to 80 years) in the electromagnetic navigation system group. In the optical navigation system group (60 cases), 54 cases were women and 6 cases were men, while in the electromagnetic navigation system group (60 cases), 51 cases were women and 9 cases were men. In all the cases, degenerative arthritis was the main cause of disease that led to TKA. The preoperative mechanical axis deviation averaged $8.4^{\circ}$ varus and was equal for both groups (Table 1).

\section{Preoperative Radiological Assessment}

Two orthopedic surgeons who had no knowledge of different groups performed radiological measurements on all patients using the picture acquiring communication system. After performing the measurements on each subject for three times, the mean

Table 1. Demographic Parameters of both Treatment Groups

\begin{tabular}{lcc}
\hline \multicolumn{1}{c}{ Parameter } & $\begin{array}{c}\text { Optical } \\
\text { navigation }\end{array}$ & $\begin{array}{c}\text { Electromagnetic } \\
\text { navigation }\end{array}$ \\
\hline No. of cases & 60 & 60 \\
Diagnosis (\%) & OA (100) & OA (100) \\
Sex (male:female) & $6: 54$ & $9: 51$ \\
Mean age (yr) & 67.5 & 68.5 \\
Preoperative mechanical axis $\left({ }^{\circ}\right)$ & Varus 8.4 & Varus 8.3 \\
\hline
\end{tabular}

OA: osteoarthritis.

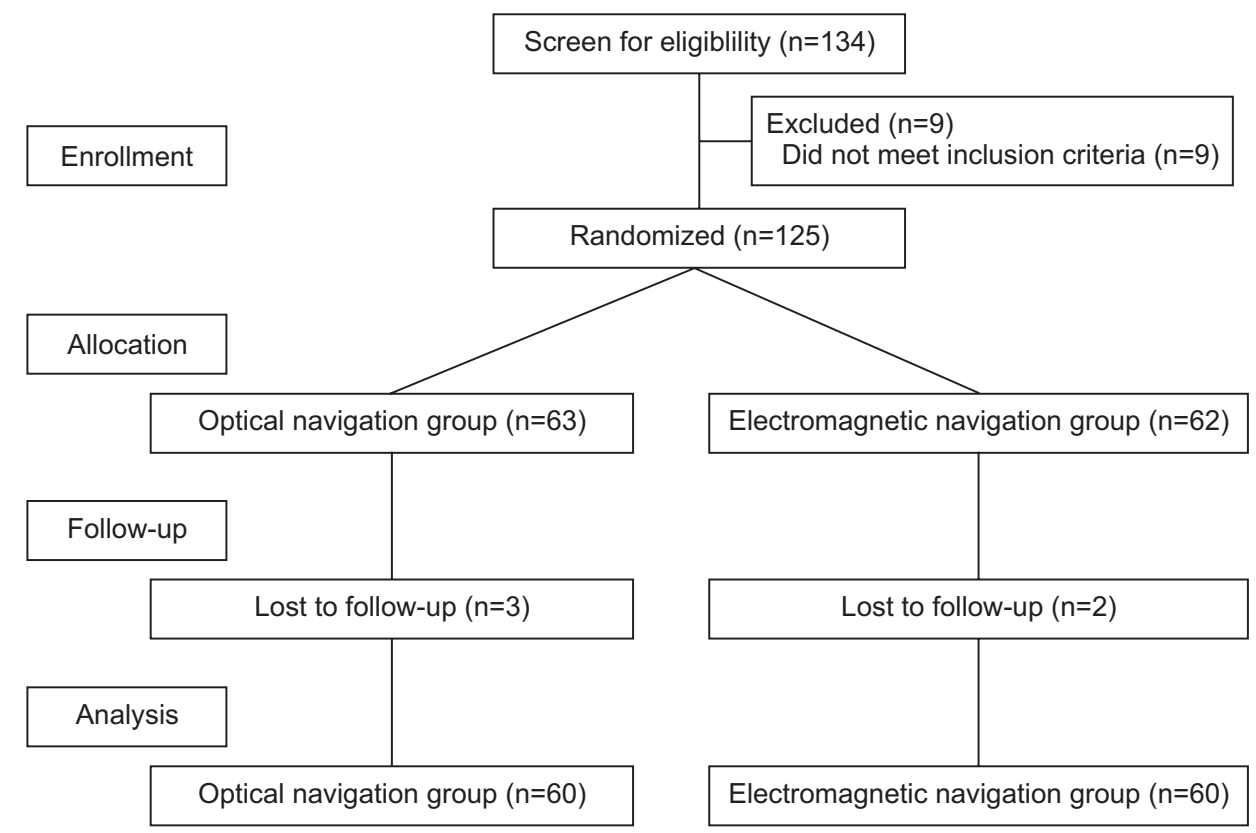

Fig. 1. Consolidated standards of reporting trials flow chart on patient enrollment. 

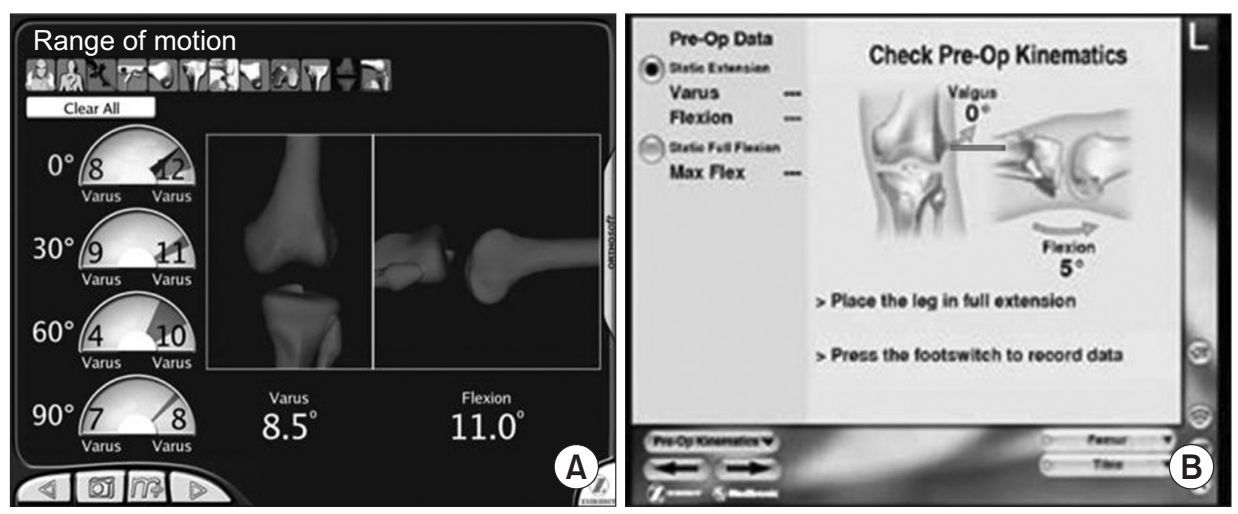

Fig. 2. Intraoperative measurement of mechanical axis deviation using the optical navigation system (A) and the electromagnetic navigation system (B).

value was determined. On the preoperative weight-bearing, fulllength orthoscanogram of both lower extremities, the deviation angle of mechanical axis was measured by measuring the angle between the line passing through the center of the femoral head and knee joint and the line passing through the center of the knee joint and talus bone.

\section{Measurements by Intraoperative Navigation System}

At the operation room, after measuring the intraoperative deviation angle of mechanical axis of the lower extremity using each navigation system, the differences between the preoperative radiological measurements and the intraoperative measurements using the navigation systems were comparatively analyzed (Fig. 2).

\section{Postoperative Radiological Assessment}

Between the 10th and the 14th day after the operation, deviation angle of the lower extremity mechanical axis and positions of the femoral and tibial implants were evaluated in the same way as the preoperative evaluation by checking the weight-bearing, fulllength orthoscanogram of both lower extremities, and anteroposterior (AP) and lateral radiographs of the knee joint. Since in our hospital, we educate and encourage TKA patients to fully extend their knee joints by their own quadriceps muscle power until at least 2 weeks after surgery, nearly all the patients could pose for orthoscanogram until postoperative day 14 . If the patient could not stand alone until day 14, orthoscanogram was performed after achieving full weight-bearing standing. The femoral and tibial component positions in the sagittal and coronal planes were assessed using the method of the Knee Society Roentgenographic Evaluation and Scoring System. The coronal inclination of the femoral component ( $\alpha$ angle) was measured as the medial angle between the mechanical axis and the bottom of the component on the AP radiographs while the sagittal inclination of the femoral component ( $\beta$ angle) was measured as the angle with the femoral shaft on the lateral views. The desired sagittal inclination was

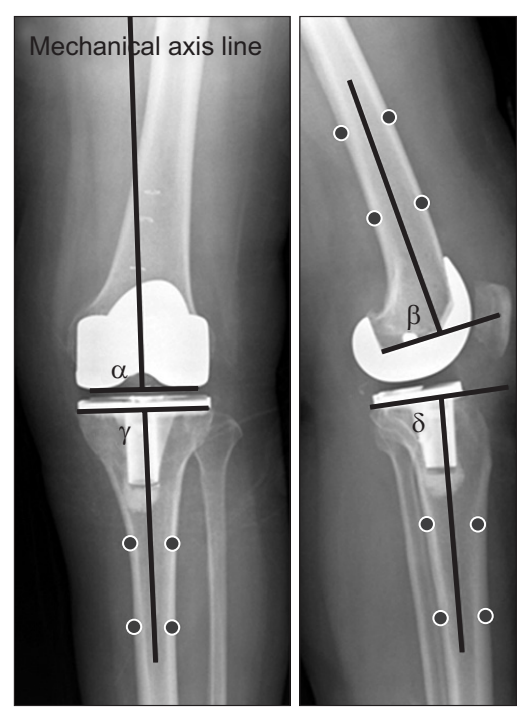

Fig. 3. Measurement of implant position angles. $\alpha$ : coronal medial inclination of femoral component, $\beta$ : sagittal posterior slope of femoral component, $\gamma$ : coronal medial inclination of tibial component, $\delta$ : sagittal posterior slope of tibial component.

defined as $90^{\circ}$. The coronal and sagittal inclinations of the tibial component $(\gamma, \delta$ angle) were assessed in a similar manner. The bisecting line of the tibial shaft was used as a reference for sagittal tibial component positioning. The desired tibial component inclination was defined as $90^{\circ}$ in the coronal plane and $86^{\circ}$ in the sagittal plane (Fig. 3).

\section{Surgical Technique}

All the operations were performed by the same surgeon using an anterior midline approach of the knee, and medial parapatellar arthrotomy was performed. Then, navigation tracker fixation was done by inserting two pins into the distal femur and proximal tibia each. For dynamic registration of the hip center, while the pelvis was fixed with both of the anterior superior iliac spines pushed against the operative table by the 2 nd assistant, the loca- 
tion of the hip joint center was identified as the average center of a large and three-planar femur-to-pelvis rotation. Registration of anatomical landmarks and axes including the femur AP axis and the medial and lateral epicondyles was performed with an instrumented pointer to define the anatomical reference frames for the femur and tibia, which provided target orientations for all relevant bone cuts and rotational alignment. The OrthoSoft optical navigation system primarily detects the mechanical axis based on the kinematic center of the joints by making motion of the hip, knee, ankle joints. Then, the secondary mathematical calibration of the mechanical axis is done depending on the information gathered by registration of the anatomical landmarks, including the distal femoral center, proximal tibial center, and medial and lateral malleolus. On the other hand, registration of the distal femoral center, proximal tibial center, and medial and lateral malleolus plus only hip motion center are necessary for the AxiEM electromagnetic navigation system to detect the mechanical axis. The hip center registration method itself is different between the two systems. The optical navigation system recognizes the hip center by spherical 3-dimensional movement of the hip joint, whereas the electromagnetic navigation system recognizes the center by 2-dimensional movement such as flexion, extension, abduction, and adduction.

Bone cutting was planned for the distal femur and proximal tibia separately with regard to the mechanical axis of each bone. The distal femoral bone cut was at $0^{\circ}$ in the coronal plane and at $3^{\circ}$ of flexion in the sagittal plane, and the proximal tibial cut was at $0^{\circ}$ in the frontal plane and $5^{\circ}$ posterior slope in the sagittal plane.
For rotational alignment, the epicondylar axis and the Whiteside line was taken for the femur. Tibial trial was left free to rotate during cycles of knee flexion and extension, and the orientation in full extension was chosen for its optimal rotational alignment. Soft tissue balancing was performed during the trial and after the final component placement according to the information from the navigation system. The tibial and femoral components were fixed with cement and patellar resurfacing was not performed in any of the cases. Posterior cruciate ligament was removed in all cases. The preoperative and postoperative mechanical axes and extent of bone resection could be evaluated using both optical and electromagnetic navigation systems. In particular, varus/valgus femoral resection, degree of flexion/extension, varus/valgus tibial resection, and tibial posterior slope could be assessed in real time during bone resection of the distal femur and the proximal tibia by placing a paddle on the resection surface (Fig. 4). After bone resection, the lower limb alignment was assessed using the navigation system with a temporary implant or an inserted spacer. After implant fixation, the alignment was evaluated and corrected in real time intraoperatively when needed. During the assessment using the navigation, authors tried to fix the body parts uniformly in routine methods to make the standardized quantification possible. Both iliac crests of the patient were compressed toward the operation table by the 2 nd assistant while we were detecting the hip center and examining the femoral landmarks in $45^{\circ}$ hip flexion. Tibial landmarks were examined with the tibia standing in vertical position. Alignment check was done with the whole lower extremity in maximal extension.
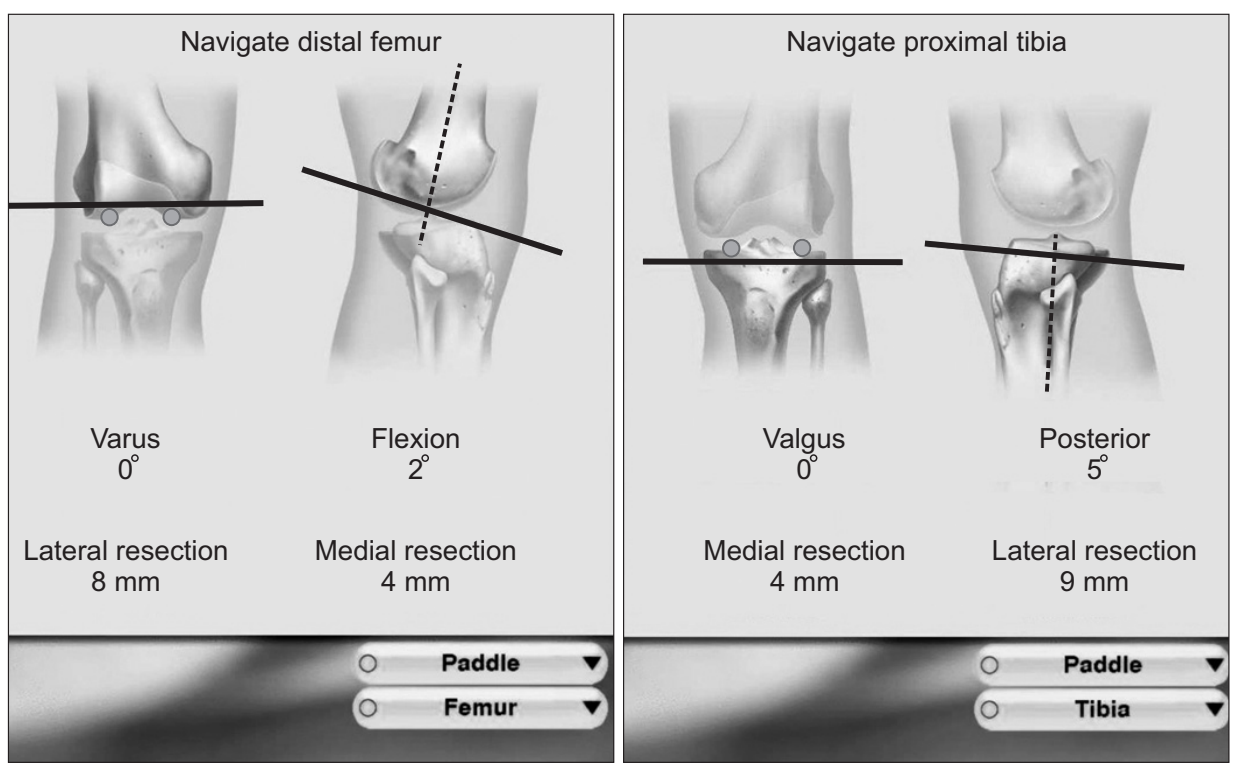

Fig. 4. A display screen in the electromagnetic navigation system during resection of the distal femur and proximal tibia (real time information). 

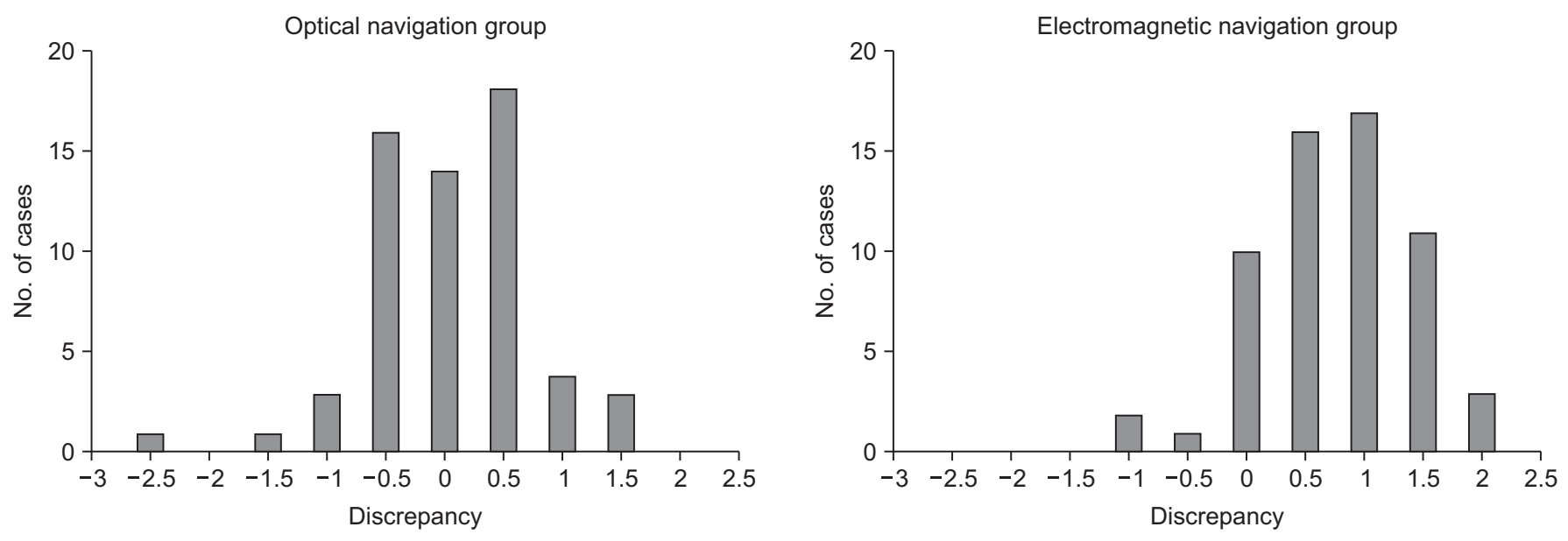

Fig. 5. Histograms of the discrepancies between the preoperative radiological measurements and the intraoperative navigation system measurements.

\section{Statistical Analysis}

In each group, difference between the mechanical axes measured on the preoperative radiographs and by the intraoperative navigation systems was comparatively analyzed through an independent $t$-test. In the postoperative analysis, radiographic values of the implant were measured by two different observers and reliability between the observers was assessed by intraclass correlation coefficient. Mann-Whitney test was used to comparatively analyze the difference of measured values of both groups. The analysis results were statistically processed with SPSS ver. 11.0 (SPSS Inc., Chicago, IL, USA) and determination of significance was based on p-value below 0.05 .

\section{Results}

\section{Comparison between the Preoperative Measurement}

of Radiological Mechanical Axis and the Intraoperative Measurement of Mechanical Axis Using Navigation Systems

The mechanical axis of the lower extremity measured by using the preoperative weight-bearing, full-length orthoscannogram was $8.4^{\circ} \pm 3.8^{\circ}$ varus in the optical navigation system group and $8.3^{\circ} \pm 3.5^{\circ}$ varus in the electromagnetic navigation system group. The mechanical axis of the lower extremity measured in the operation room by using each navigation system showed $8.2^{\circ} \pm 3.0^{\circ}$ varus in the optical navigation system group and $8.7^{\circ} \pm 2.8^{\circ}$ varus in the electromagnetic navigation system group. The difference between the mechanical axes measured on the preoperative radiographs and by the intraoperative navigation system was $0.2^{\circ} \pm 0.8^{\circ}$ valgus in the optical navigation system group and $0.4^{\circ} \pm 0.7^{\circ}$ varus in the electromagnetic navigation system group. Histograms of the discrepancies between the preoperative radiological measurement and the intraoperative navigation system
Table 2. Mechanical Axis Deviation of both Treatment Groups Measured by Orthoroentgenogram and Navigation Systems

\begin{tabular}{lrrr}
\hline \multicolumn{1}{c}{ Variable } & $\begin{array}{c}\text { Optical } \\
\text { navigation }\end{array}$ & $\begin{array}{c}\text { Electromagnetic } \\
\text { navigation }\end{array}$ & p-value \\
\hline $\begin{array}{l}\text { Mechanical axis deviation }\left(^{\circ}\right) \\
\text { Orthoroentgenogram }\end{array}$ & Varus $8.4 \pm 3.8$ & Varus 8.3 \pm 3.5 & \\
Navigation system & Varus $8.2 \pm 3.0$ & Varus $8.7 \pm 2.8$ & \\
Differences & Valgus $0.2 \pm 0.8$ & Varus $0.4 \pm 0.7$ & $>0.05$ \\
\hline
\end{tabular}

Values are presented as mean \pm standard deviation.

measurement showed normal distribution in both the optical and electromagnetic navigation groups. When the acceptable range of discrepancy was set as within $2^{\circ}$ standard deviation range, there was 1 outlier in the optical navigation group and none in the electromagnetic navigation group (Fig. 5).

The discrepancy between the preoperative radiological measurement and the intraoperative measurement appeared to be more varus by $0.6^{\circ}$ in the electromagnetic navigation system group than that in the optical navigation system group, but the intergroup difference was not statistically significant $(\mathrm{p}>0.05)$ (Table 2).

\section{Postoperative Radiological Results}

In the optical navigation system group, the mechanical axis measured by observer 1 was corrected from $8.4^{\circ} \pm 3.7^{\circ}$ varus preoperatively to $0.9^{\circ} \pm 1.2^{\circ}$ varus postoperatively. The coronal inclination of the femoral component ( $\alpha$ angle) was $89.3^{\circ} \pm 2.8^{\circ}$ and the coronal inclination of the tibial component ( $\gamma$ angle) was $88.5^{\circ} \pm 1.5^{\circ}$, while the sagittal inclination of the femoral component ( $\beta$ angle) was $89.8^{\circ} \pm 1.0^{\circ}$ and that of the tibial component $(\delta$ angle) was $86.6^{\circ} \pm 1.4^{\circ}$. The mechanical axis measured by observer 
Table 3. Radiological Results of Implant Position after Total Knee Arthroplasty and Interobserver Reliability

\begin{tabular}{|c|c|c|c|c|c|c|}
\hline \multirow{2}{*}{ Angle $\left({ }^{\circ}\right)$} & \multicolumn{3}{|c|}{ Optical navigation } & \multicolumn{3}{|c|}{ Electromagnetic navigation } \\
\hline & Observer 1 & Observer 2 & ICC & Observer 1 & Observer 2 & ICC \\
\hline$\alpha$ & $89.3 \pm 2.8$ & $89.5 \pm 2.4$ & 0.9839 & $89.6 \pm 3.2$ & $89.9 \pm 2.9$ & 0.9851 \\
\hline$\beta$ & $89.8 \pm 1.0$ & $89.5 \pm 1.2$ & 0.9721 & $89.3 \pm 1.2$ & $89.8 \pm 1.3$ & 0.8995 \\
\hline$\gamma$ & $88.5 \pm 1.5$ & $89.0 \pm 1.7$ & 0.9116 & $88.5 \pm 1.8$ & $89.1 \pm 2.0$ & 0.9552 \\
\hline$\delta$ & $86.6 \pm 1.4$ & $86.8 \pm 1.3$ & 0.9085 & $86.8 \pm 2.0$ & $86.4 \pm 1.2$ & 0.9441 \\
\hline
\end{tabular}

Values are presented as mean \pm standard deviation. $p$-value $<0.001$.

ICC: intraclass correlation coefficient.

2 was corrected from $8.3^{\circ} \pm 3.5^{\circ}$ varus preoperatively to $0.8^{\circ} \pm 1.0^{\circ}$ varus postoperatively. The angles of component inclination were measured by observer 2 as a angle of $89.5^{\circ} \pm 2.4^{\circ}, \gamma$ angle of $89.0^{\circ} \pm 1.7^{\circ}, \beta$ angle of $89.5^{\circ} \pm 1.2^{\circ}$, and $\delta$ angle of $86.8^{\circ} \pm 1.3^{\circ}$.

In the electromagnetic navigation system group, the mechanical axis of the lower extremity measured by observer 1 was corrected from $8.3^{\circ} \pm 3.6^{\circ}$ varus preoperatively to $0.9^{\circ} \pm 1.0^{\circ}$ varus postoperatively, and the angles of component inclination were measured as $\alpha$ angle of $89.6^{\circ} \pm 3.2^{\circ}, \gamma$ angle of $88.5^{\circ} \pm 1.8^{\circ}, \beta$ angle of $89.3^{\circ} \pm 1.2^{\circ}$, and $\delta$ angle of $86.8^{\circ} \pm 2.0^{\circ}$. The mechanical axis measured by observer 2 was corrected from $8.4^{\circ} \pm 3.5^{\circ}$ varus preoperatively to $0.7^{\circ} \pm 0.9^{\circ}$ varus postoperatively, and the angles of component inclination were measured as a angle of $89.9^{\circ} \pm 2.9^{\circ}, \gamma$ angle of $89.1^{\circ} \pm 2.0^{\circ}, \beta$ angle of $89.8^{\circ} \pm 1.3^{\circ}$, and $\delta$ angle of $86.4^{\circ} \pm 1.2^{\circ}$. Mechanical axis deviations and all the angles of the femoral and tibial components on the sagittal and coronal planes measured by observer $1 \& 2$ in the optical and the electromagnetic navigation system groups showed high intraclass correlation coefficients (Table 3), which, in turn, can be interpreted that the possibility of interobserver error between the two observers is statistically low.

\section{Discussion}

Though there are diverse navigation systems including the optical navigation system, electromagnetic navigation system, and navigation system using ultrasound, few comparative clinical studies have been conducted on the precision of different navigation systems ${ }^{4,7-10)}$. And, there are a number of factors that possibly affect the precision of the navigation system, such as the difference in tracking devices and registration method of anatomical landmarks and motion centers. The existence of thick soft tissue coverings also can be an obstacle to the navigation system.

So, we tried to analyze and compare the precision of the optical navigation system and electromagnetic navigation system in the real operating condition. Discrepancy between the preoperative mechanical axis measurement using radiographs and intraopera- tive mechanical axis measurement using the navigation system was calculated. The discrepancy was $0.2^{\circ} \pm 0.8^{\circ}$ valgus in the optical navigation system group and $0.4^{\circ} \pm 0.7^{\circ}$ varus in the electromagnetic navigation system group. Although, the discrepancy was $0.6^{\circ}$ more varus in the electromagnetic navigation system group, the difference was not statistically significant between the two groups.

Compared with our results, other literatures dealing with the precision of the navigation system reported various results. Song et al. ${ }^{6}$ reported that, in 20 cases of TKA, the mechanical axis of the lower extremities measured by the intraoperative optical navigation was $9.02^{\circ}$ varus, while that measured by the electromagnetic navigation system was $10.25^{\circ}$ varus. The mechanical axis was more varus when measured using the electromagnetic navigation system, but statistically significant difference was not noted between the two systems. They reported that the accuracy and reproducibility of the optical navigation system were higher than those of the electromagnetic navigation system. Moreover, they reported that in cases of intentional erroneous registration of anatomical landmarks, measurements of the mechanical axis of these two navigation systems were affected thereby. They suggested precaution is particularly required in the whole process of registration using the electromagnetic navigation system because the variation range is broader in the system. Song et al. ${ }^{6}$ compared the intraoperative mechanical axis deviation using the optical navigation and the electromagnetic navigation in the same patient. In contrast, we assessed the precision of the navigation system by comparing the preoperative mechanical axis deviation measured with orthoroentgenogram and intraoperative mechanical axis deviation measured using the navigation system. And the patients were divided into two separate groups for optical and electromagnetic navigation. We could analyze a relatively large number of cases because our study did not include any potentially hazardous procedures such as attaching two different navigation systems sequentially. It is our understanding that our results demonstrated the equal precision of the optical and elec- 
tromagnetic navigation systems with more power based on the greater number of cases than that in other studies.

Steihl and Heck ${ }^{11)}$ compared the precision of the optical and electromagnetic navigation systems using a cadaver in a standard operating room, and reported that both navigation systems showed satisfactory results in the mechanical axis measurement, but did not show satisfactory results in the measurement of the transepicondylar axis and AP axis. However, they reported that in the case of electromagnetic navigation system, its accuracy may be affected by electromagnetic forces in the operation room environment. As to the optical navigation system using infrared, Pitto et al. ${ }^{5)}$ reported under $0.5^{\circ}$ of average error for the normal alignment and under $1.0^{\circ}$ of average error for the abnormal alignment. Yau et al. ${ }^{12)}$ reported maximum error range of $1.32^{\circ}$ in the coronal plane and $4.17^{\circ}$ in the sagittal plane in the cadaveric study. There are also reports regarding the electromagnetic navigation system. Hummel et al. ${ }^{8}$ reported that Aurora navigation was relatively precise with an error range of $0.97 \mathrm{~mm}$ in distance and rotational error of $0.2^{\circ}$ to $0.91^{\circ}$, but they also reported that electromagnetic navigation measurement could be distorted by 400 series stainless steel. Schicho et al. ${ }^{10)}$ reported that the metal retractors or drilling devices or ultrasonographic probes can cause electromagnetic navigation errors, but they also reported lower errors with the more recent navigation system. According to the abovementioned reports, the electromagnetic navigation is affected by the metal artifacts, but the influence has been decreasing as the navigation system improves.

We also experienced practical problems with the optical navigation system. The trackers of the optical navigation system are relatively huge, disrupting the operative field to secure the line of site' compared to the electromagnetic navigation system; thus, we had to retract more medial soft tissue and often held the leg externally rotated for the better 'line of site', which are all possibly related to the errors in registration and operation. In our study, the discrepancy between the preoperative radiological measurement of the mechanical axis and the intraoperative measurement using the navigation system appeared to be relatively more varus in the electromagnetic navigation group, although both groups showed no statistically significant difference. The postoperative mechanical axis deviation and the positions of femoral and tibial components on the coronal and sagittal planes also showed no statistically significant difference between the two groups. And the reason for the varus tendency requires further analysis of the factors related to TKA using navigation system.

There are some limitations in our study. First, the exclusion and inclusion criteria of this study were dilemmatic. Navigation systems in TKA are especially helpful in patients with clinical manifestation of severe joint deformity. However, we could not help excluding the patients with a varus or valgus deformity of more than $15^{\circ}$ and those with a posttraumatic or postoperative deformity, which may limit the clinical significance of this study. Second, the preoperative radiographs were taken in a weightbearing position, whereas the intraoperative measurements were performed with the patient lying on the table. This could have contributed to the difference in the mechanical axis measured from the radiograph and navigation system, which should not be overlooked and warrants further investigation.

\section{Conclusions}

Both optical and electromagnetic navigation systems have demonstrated high precision and consistency in terms of mechanical axis deviation and measurement of implant position. We believe our results provide answers to the surgeons who are questioning about the superiority of different navigation systems. In TKA using navigation systems, the accuracy of anatomical registration process should be confirmed and the preoperative joint deformity and the degree of instability should be identified before operation. In addition, efforts should be made to eliminate any possible causes of error related to computer equipment, such as pin movement, as much as possible.

\section{Conflict of Interest}

No potential conflict of interest relevant to this article was reported.

\section{Acknowledgments}

This work was supported by a 2-Year Research Grant of Pusan National University.

\section{References}

1. Lotke PA, Ecker ML. Influence of positioning of prosthesis in total knee replacement. J Bone Joint Surg Am. 1977;59:779.

2. Moreland JR. Mechanisms of failure in total knee arthroplasty. Clin Orthop Relat Res. 1988;(226):49-64.

3. Ritter MA, Faris PM, Keating EM, Meding JB. Postoperative alignment of total knee replacement. Its effect on survival. Clin Orthop Relat Res. 1994;(299):153-6. 
4. Amin DV, Kanade T, DiGioia AM 3rd, Jaramaz B. Ultrasound registration of the bone surface for surgical navigation. Comput Aided Surg. 2003;8:1-16.

5. Pitto RP, Graydon AJ, Bradley L, Malak SF, Walker CG, Anderson IA. Accuracy of a computer-assisted navigation system for total knee replacement. J Bone Joint Surg Br. 2006; 88:601-5.

6. Song EK, Seon JK, Park SJ, Yoon TR. Accuracy of navigation: a comparative study of infrared optical and electromagnetic navigation. Orthopedics. 2008;31(10 Suppl 1).

7. Chen TK, Abolmaesumi P, Pichora DR, Ellis RE. A system for ultrasound-guided computer-assisted orthopaedic surgery. Comput Aided Surg. 2005;10:281-92.

8. Hummel J, Figl M, Birkfellner W, Bax MR, Shahidi R, Maurer CR Jr, Bergmann H. Evaluation of a new electromagnetic tracking system using a standardized assessment protocol. Phys Med Biol. 2006;51:N205-10.
9. Khadem R, Yeh CC, Sadeghi-Tehrani M, Bax MR, Johnson JA, Welch JN, Wilkinson EP, Shahidi R. Comparative tracking error analysis of five different optical tracking systems. Comput Aided Surg. 2000;5:98-107.

10. Schicho K, Figl M, Donat M, Birkfellner W, Seemann R, Wagner A, Bergmann H, Ewers R. Stability of miniature electromagnetic tracking systems. Phys Med Biol. 2005;50: 2089-98.

11. Stiehl JB, Heck DA. Six sigma analysis of computer-assisted surgery tracking protocols in TKA. Clin Orthop Relat Res. 2007;464:105-10.

12. Yau WP, Leung A, Chiu KY, Tang WM, Ng TP. Intraobserver errors in obtaining visually selected anatomic landmarks during registration process in nonimage-based navigationassisted total knee arthroplasty: a cadaveric experiment. J Arthroplasty. 2005;20:591-601. 